\title{
Soft-x-ray laser interferometry of a plasma with a tabletop laser and a Lloyd's mirror
}

\author{
J. J. Rocca, C. H. Moreno, M. C. Marconi, and K. Kanizay \\ Department of Electrical Engineering, Colorado State University, Fort Collins, Colorado 80523
}

Received November 17, 1998

\begin{abstract}
We report what is believed to be the first demonstration of soft-x-ray interferometry of a plasma with a tabletop soft-x-ray laser. A Lloyd's mirror interferometer was used in combination with a very compact $\lambda=46.9 \mathrm{~nm}$ capillary-discharge-pumped laser to map the electron density in the cathode region of a pinch plasma. (C) 1999 Optical Society of America

OCIS codes: $140.7240,170.7440,120.3180$.
\end{abstract}

Interferometry using optical lasers is a powerful technique for the diagnostics of plasmas that provides time-resolved two-dimensional maps of the electron density. However, free-free absorption and refraction of the probe beam limit the maximum electron density, plasma size, and plasma density gradients that can be probed with optical wavelengths. The development of soft-x-ray lasers has opened the possibility of extending plasma interferometry to shorter wavelengths, significantly expanding the range of plasma parameters that can be probed. Recently, a 15.5-nm Ne-like Y laser pumped by the Nova laser at Lawrence Livermore National Laboratories was used in combination with a skewed Mach-Zehnder interferometer to probe very high-density laser-created plasmas. ${ }^{1,2}$ The amplitude-division interferometer used in those pioneering experiments was implemented by use of thin multilayer beam splitters and multilayer-coated mirrors developed for that wavelength. The advent of gain-saturated discharge-pumped tabletop soft-x-ray lasers $^{3,4}$ and the development of several saturated ${ }^{5}$ or nearly saturated ${ }^{6,7}$ soft-x-ray lasers pumped by relatively compact optical lasers will allow the probing of a great variety of dense plasmas. However, multilayer beam splitters cannot be developed for the wavelengths corresponding to some of these lasers at present, owing to the lack of materials with adequate optical constants. Alternatively, amplitude-division interferometers based on diffraction gratings ${ }^{8}$ and wavefront-division interferometers ${ }^{9,10}$ have been discussed. Recently, a Fresnel bimirror wave-front-division soft$\mathrm{x}$-ray interferometer was demonstrated ${ }^{10}$ and was used in combination with a 21.2-nm Ne-like $\mathrm{Zn}$ laser to probe surface defects induced by an intense electric field. ${ }^{11}$

In this Letter we report what we believe to be the first use of a tabletop soft-x-ray laser in plasma interferometry. We have utilized a very compact $\lambda=$ $46.9 \mathrm{~nm}$ capillary-discharge-pumped tabletop soft-xray laser in combination with a Lloyd's mirror ${ }^{12}$ to probe the cathode region of a pinch plasma with subnanosecond time resolution. The maximum plasma electron density $n_{e}$ and size $L$ that can be probed with this laser are significantly larger than those accessible with the fourth harmonic of a Nd:YAG laser at $\lambda=265 \mathrm{~nm}$ (free-free absorption, $\propto n_{e}{ }^{2} L$, is signifi- cantly smaller at $46.9 \mathrm{~nm}$, e.g., $\approx 36$ times smaller for a plasma with an electron temperature $T_{e}=100 \mathrm{eV}$ ). Moreover, larger plasma gradients can be probed at this short wavelength owing to the reduction of $\approx 30$ times in the refraction angle. A Lloyd's mirror is the simplest possible wave-front-division interferometer, and because it is based on a grazing-incidence reflection it is particularly well suited for soft-x-ray interferometry. To implement this interferometer scheme one positions a mirror at a grazing-incidence angle $\delta$ to intercept part of the beam emitted by a coherent source. As illustrated in Fig. 1, the interference arises from the superposition of the fraction of the beam reflected in the mirror and the beam that propagates directly from the source. The interference pattern can be described as resulting from the interference of the radiation emitted by a point source $\mathrm{S}$ and its virtual image $\mathrm{S}^{\prime} .{ }^{12}$ We formed $\mathrm{S}$ by focusing the soft-x-ray laser beam with a concave mirror. The interfringe separation $\Delta I$ at the detector plane is

$$
\Delta I=\frac{(b+c) \lambda}{d}=\frac{(b+c) \lambda}{2 b \sin \delta}
$$

where $b$ is the distance between $\mathrm{S}$ and a generic location on the Lloyd's mirror, $c$ is the distance from that location to the image plane, and $d=2 b \sin \delta$ is the distance between the two interfering sources, $\mathrm{S}$ and $\mathrm{S}^{\prime}$. The object under study (in our case the plasma) is placed in the laser beam path in such a way that it intercepts only part of the beam, leaving the rest unchanged for use as the reference beam.

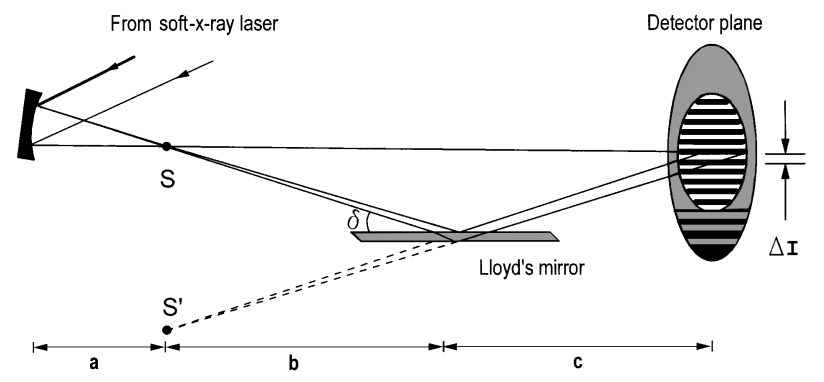

Fig. 1. Schematic diagram of a Lloyd's mirror interferometer.

(C) 1999 Optical Society of America 
The object location with respect to the curved mirror and the distance between the mirror and the detector are chosen so that a real image with the desired magnification at the detector plane is obtained.

The setup of the entire experiment is shown in Fig. 2. The 46.9-nm soft-x-ray laser beam was produced by excitation of a 4-mm-diameter 16.4-cm-long capillary channel in polyacetal filled with $\sim 600 \mathrm{mTorr}$ of Ar, with a fast current pulse of $\approx 37-\mathrm{kA}$ peak amplitude and a 72-ns first half-cycle duration. ${ }^{4}$ The laser emits pulses of up to $25 \mu \mathrm{J}$ in energy and $0.6-0.7-n s$ duration. Under these discharge conditions the laser beam has a divergence of $\approx 5 \mathrm{mrad}$ and is spatially coherent within a cone having a full angle of at least 0.5 mrad. ${ }^{13}$ We selected the distance between the laser and the plasma, $\approx 2.4 \mathrm{~m}$, to have a spatial-coherence length larger than $1.2 \mathrm{~mm}$ at the plasma location. We used a spherical mirror having a radius of curvature $R=60 \mathrm{~cm}$ at near-normal incidence to image the plasma on the detector and to generate focalized source S. An auxiliary flat mirror relayed the image onto the detector. The mirrors were both fabricated by coating of superpolished substrates with $\mathrm{Sc}-\mathrm{Si}$ multilayers. ${ }^{14}$ The reflectivity of these multilayer mirrors is $\approx 34 \%$ for normal incidence at $46.9 \mathrm{~nm}$, with a bandwidth of $\approx 4 \mathrm{~nm} .{ }^{14}$ We fabricated the Lloyd's mirror by cleaving a polished Si wafer to obtain a strip $2.5 \mathrm{~cm}$ wide and $18 \mathrm{~cm}$ long. The detector was composed of a microchannel plane (MCP), an Al-coated phosphor screen, and an image intensifier followed by a $1024 \times 1024 \mathrm{CCD}$ array. Gating the microchannel plate during $\approx 5 \mathrm{~ns}$ in coincidence with the arrival of the laser pulse on the detector reduced the background signal caused by the plasma self-emission. The distance from the spherical mirror to the center of the Lloyd's mirror was $43.7 \mathrm{~cm}$. The distance from the center of the Lloyd's mirror to the detector was $632 \mathrm{~cm}$. The plasma was placed at $31.4 \mathrm{~cm}$ from the curved mirror and imaged with a magnification of $21.5 \times$ onto the detector. We placed the Lloyd's mirror at an incidence angle of $\approx 1.4 \mathrm{mrad}$ to obtain an interfringe separation corresponding to a distance of $38 \mu \mathrm{m}$ at the object plane.

The plasma that was studied was the cathode region of a pulsed discharge. The discharge was produced between two main electrodes, a grounded planar stainless-steel anode and a $\mathrm{Cu}$ cathode shaped in the form of a 5-mm-long knife edge (-HV in Fig. 2). The electrode separation was $4 \mathrm{~mm}$. A predischarge between a third auxiliary electrode $(+\mathrm{HV}$ in Fig. 2) and the anode produced through an evacuated polyacetal capillary channel injected material ablated from the walls of the channel into the space between the main electrodes. A pinch plasma was subsequently generated in the proximity of the cathode by an $\approx 30$-kA 1.8- $\mu \mathrm{s}$ half-period discharge-current pulse produced by discharge of a $3.2-\mu \mathrm{F}$ capacitor across the main electrodes through a triggered spark gap. A vacuum photodiode was used to monitor the VUV and soft$\mathrm{x}$-ray radiation emitted by the plasma in every shot. The time at which the pinch occurred was identified by a pronounced peak of $\approx 150$-ns duration in the photodiode signal. Emission spectroscopy showed that the plasma contained $\mathrm{C}$ and $\mathrm{O}$ ions (ionized up to $\mathrm{O}$ v) resulting from material ablated by the precursor discharge and $\mathrm{Cu}$ ions arising from vaporization of the cathode. The spectra were dominated by lines of $\mathrm{O}$ IV-O V and contained lines of $\mathrm{Cu}$ III and $\mathrm{Cu}$ IV. The extent of the plasma along the path of the probe beam (parallel to the cathode's edge) was estimated to be $L=(1.9 \pm 0.3) \mathrm{mm}$ from the size of the soft-x-ray emitting region of the plasma, measured in an auxiliary plasma-imaging experiment.

Figure 3 shows a set of two interferograms. The top image corresponds to a reference shot where no plasma was present. The shadow of the cathode electrode of the discharge is observed at the right of the interferogram. The interference fringes created by the Lloyd's mirror cover the central part of the image and are distinguished as equally spaced fringes with a visibility of $\approx 40 \%$ in the central zone. The unevenly spaced fringes at the bottom of the interferograms have significantly lower visibility and are caused by diffraction of the laser beam on the edge of Lloyd's mirror. The bottom part of Fig. 3 is an interferogram of the plasma obtained $160 \mathrm{~ns}$ after the time of maximum soft-x-ray emission by the plasma. The shadow of the cathode is no longer clearly visible because of the plasma selfemission; nevertheless, the fringe visibility remains good. The distortion of the fringes reveals the presence of a high-density plasma close to the cathode tip.

The plasma density was computed from the measured fringe shifts, assuming that the plasma is uniform along the probe path. The elongated shape of the plasma along the cathode's edge and the relatively uniform intensity of soft-x-ray self-emission along the probe path provide some justification for this approximation. The fringe shift, $\Delta \Phi$, is determined by

$$
\Delta \Phi=\int_{0}^{L} \frac{\Delta \eta}{\lambda} \mathrm{d} x \approx \frac{L}{2 \lambda} \frac{n_{e}}{n_{e c}}
$$

where $x$ is measured along the probe beam. The index of refraction of the plasma $\eta$ relates to the electron density, $n_{e}$, as $\eta=\left(1-n_{e} / n_{e c}\right)^{1 / 2}$, where

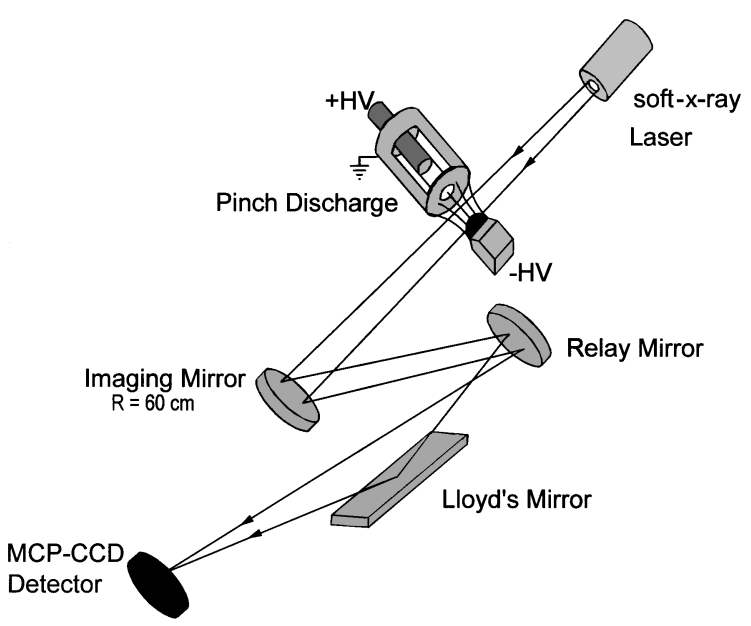

Fig. 2. Schematic representation of the experimental setup utilized in the plasma interferometry experiment. 


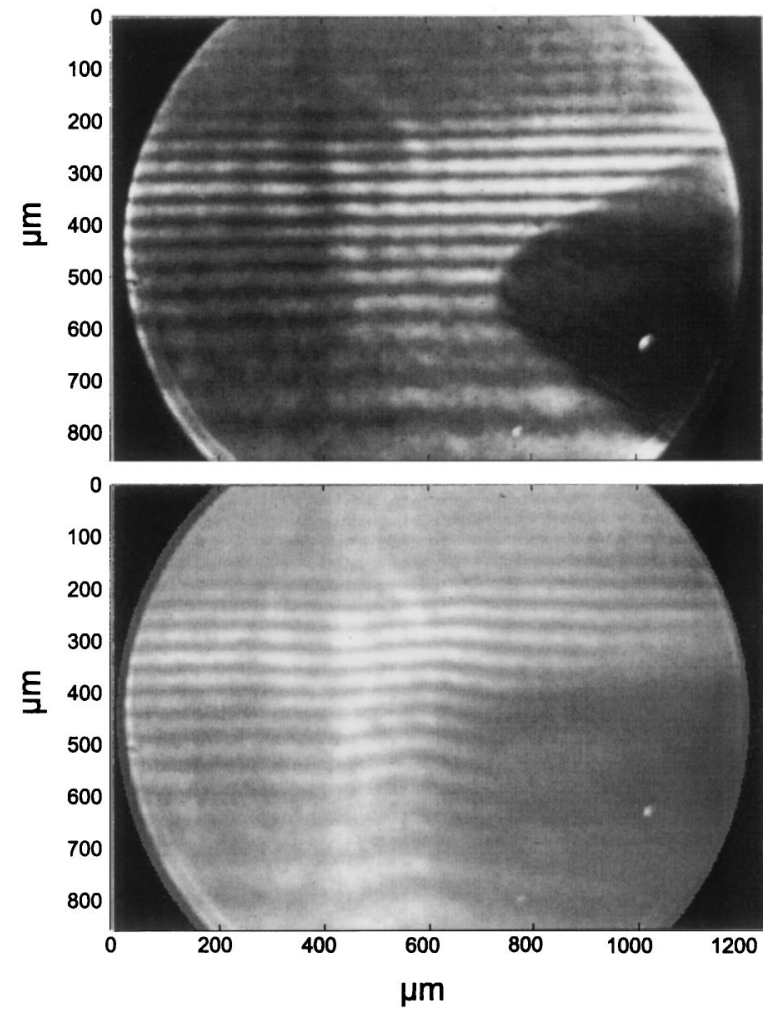

Fig. 3. Top, reference soft-x-ray interferogram (no plasma present). The shadow of the cathode electrode can be seen at the right. (b) Soft-x-ray interferogram of the pinch plasma. Fringe shifts owing to the plasma can be observed.

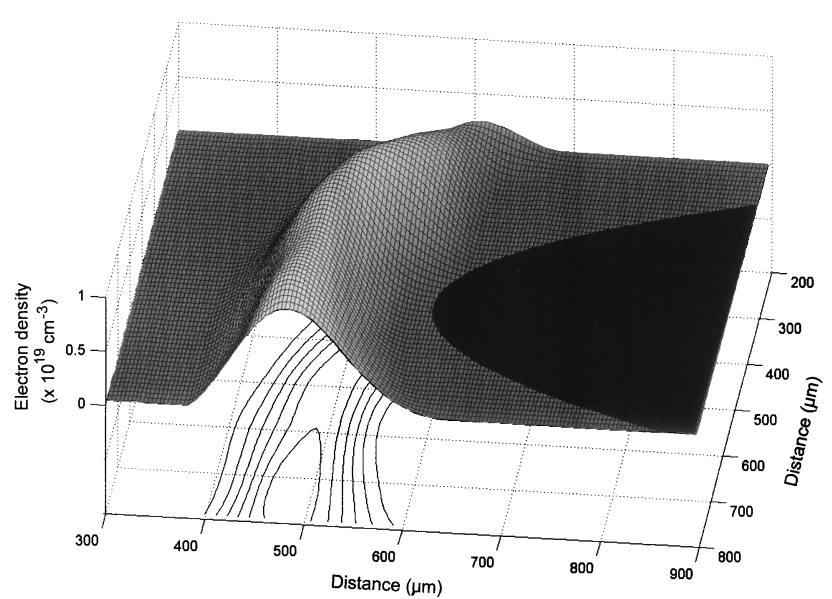

Fig. 4. Two-dimensional electron-density profile computed from the bottom interferogram of Fig. 3. The dark area at the right corresponds to a cross section of the cathode electrode (the cathode's knife edge is parallel to the vertical direction).

$n_{e c}=5 \times 10^{23} \mathrm{~cm}^{-3}$ is the critical density for 46.9-nm radiation. Figure 4 shows the twodimensional electron-density profile resulting from the analysis of Fig. 3. The denser part of the plasma concentrates mostly in a region within $300 \mu \mathrm{m}$ of the cathode. The peak measured value of the electron density is $(0.95 \pm 0.30) \times 10^{19} \mathrm{~cm}^{-3}$ and occurs at a distance of $\approx 120 \mu \mathrm{m}$ from the cathode edge.

In summary, we have realized what we believe to be the first demonstration of soft-x-ray interferometry utilizing a tabletop laser. This is also the first time to our knowledge that a wave-front-division soft-x-ray interferometer has been used for plasma diagnostics. These results may lead to the use of tabletop soft-x-ray lasers in probing a great variety of dense plasmas.

This work was supported by U.S. Department of Energy grant DE-FG03-98DP00208. The development of the laser was supported by the National Science Foundation. We acknowledge the support of grant RP1-240 from the U.S. Civilian Research and Development Foundation for the Independent States of the Former Soviet Union (CRDF) for a collaboration with A. Vinogradov and co-workers for the development of the multilayer mirrors. J. J. Rocca's e-mail address is rocca@eng.colostate.edu.

\section{References}

1. L. B. Da Silva, T. W. Barbee, Jr., R. Cauble, P. Celliers, D. Ciarlo, S. Libby, R. A. London, D. Matthews, S. Mrowka, J. C. Moreno, D. Ress, J. E. Trebes, A. S. Wan, and F. Weber, Phys. Rev. Lett. 74, 3991 (1995).

2. A. S. Wan, T. W. Barbee, Jr., R. Cauble, P. Celliers, L. B. Da Silva, J. C. Moreno, P. W. Rambo, G. F. Stone, J. E. Trebes, and F. Weber, Phys. Rev. E 55, 6293 (1997).

3. J. J. Rocca, D. P. Clark, J. L. A. Chilla, and V. N. Shlyaptsev, Phys. Rev. Lett. 77, 1476 (1996).

4. B. R. Benware, C. H. Moreno, D. Burd, and J. J. Rocca, Opt. Lett. 22, 796 (1997).

5. R. Tomassini, F. Löwenthal, and J. E. Balmer, "X ray lasing and saturation in Ni-like ions at low pump power," in Proceedings of the Sixth International Conference on X-Ray Lasers (to be published).

6. P. V. Nickles, V. N. Shlyaptsev, M. Kalashnikov, M. Schnurer, I. Will, and W. Sander, Phys. Rev. Lett. 78, 2748 (1977).

7. J. Dunn, A. L. Osterheld, R. Shepherd, W. E. White, V. N. Shlyaptsev, and R. E. Stewart, Phys. Rev. Lett. 80, 2825 (1998).

8. J. L. A. Chilla, J. J. Rocca, O. E. Martinez, and M. C. Marconi, Opt. Lett. 21, 955 (1996).

9. J. Svatos, D. Joyeux, D. Phalippou, and F. Polack, Opt. Lett. 18, 1367 (1993).

10. F. Albert, D. Joyeux, P. Jaeglé, A. Carillon, J. P. Chauvineau, G. Jamelot, A. Klishnick, J. C. Lagron, D. Phalippou, D. Ros, S. Sebban, and P. Zeitoun, Opt. Commun. 142, 184 (1997).

11. Ph. Zeitoun, F. Albert, P. Jaeglé, D. Joyeux, M. Bossoukaya, A. Carrillon, S. Hubert, G. Jamelot, A. Klishnick, D. Phalippov, J. C. Lagron, D. Ross, S. Sebban, and A. Zeitoun-Fakiris, Nucl. Instrum. Meth. Phys. Res. A 416, 189 (1998).

12. M. Born and E. Wolf, eds., Principles of Optics, 5th ed. (Pergamon, New York, 1975).

13. M. C. Marconi, J. L. A. Chilla, C. H. Moreno, B. R. Benware, and J. J. Rocca, Phys. Rev. Lett. 79, 2799 (1997).

14. Yu. A. Uspenskii, V. E. Lavashov, A. V. Vinogradov, A. I. Fedorenko, V. V. Kondratenko, Yu. P. Pershin, E. N. Zubarev, and V. Yu Fedotov, Opt. Lett. 23, 771 (1998). 